\title{
INTELLIGENT DECISION-MAKING SYSTEM FOR AUTONOMOUS ROBOTS
}

\author{
ZDZISŁAW KOWALCZUK, MichaŁ CZUBENKO
}

\author{
Decision Systems Department, Faculty of Electronics, Telecommunications and Informatics \\ Gdańsk University of Technology, Narutowicza 11/12, 80-233, Gdańsk, Poland \\ e-mail: kova@pg.gda.pl m.czubenko@gmail.com
}

\begin{abstract}
The paper gives an account of research results concerning a project on creating a fully autonomous robotic decisionmaking system, able to interact with its environment and based on a mathematical model of human cognitive-behavioural psychology, with some key elements of personality psychology included. The principal idea of the paper is focused on the concept of needs, with a certain instrumental role of emotions.
\end{abstract}

Keywords: autonomous robots, artificial intelligence, decision making systems, psychological processes.

\section{Introduction}

Creating devices which can freely move in an environment unapproachable for human beings (for instance, in the lack of oxygen) has long been a principal subject of robotics. Using a remote control module is the basic solution to such problems. Still, there are several important factors limiting this approach like visibility, communication distance, delay, etc. An alternative is to use autonomous modules, which can take over control at the request of a system operator, or are completely autonomous entities (Kowalczuk and Czubenko, 2009).

Their biggest disadvantage lies in a generally weak adaptation to a changing environment. There are various autonomous units, which are able to operate on foreign planets, deserts, cities, or swampy grounds, and so forth. The design problems are not only in their physical construction, but also in their algorithms of behaviour. In particular, instead of specializing the autonomous entities to a precisely known terrain, it can be advisable to increase their adaptation to a given varying environment.

Adaptation can be based on various ideas taken from nature (like genetic algorithms, learning neural networks, etc.). The concept of this work consists in utilizing models of psychology of living creatures (humans, in particular) to cope with the issue of adaptation to the environment of autonomous robots (Kowalczuk and Czubenko, 2010b).

From the current viewpoint of technical sciences, and, in particular, from the automatic control and robotics perspectives, it can be observed that the history of science (and, in particular, the development of cybernetics and ar- tificial intelligence) can be considered an instrumental 'reaction' to the lack of precise mathematical solutions. As a result, scientists have been directed to research and observation of the nature, including human beings and their cognition. Note that psychological exploration of human cognition is practically performed by experimenting with mammals, like dolphins, rats, rabbits, squirrels, etc. In effect, it has been proved that mammals have emotions! However, human beings represent the highest form of life, and have the most well developed auto-adaptation system. Therefore, our models and solutions for robotic development have been built on the basis of human psychology and its relevant terminology. A 'simple' application of this methodology can be found in the attractive idea of a perfect soldier performing autonomously a certain task.

Accordingly, many scientific projects concern the modelling of human psychology. They focus on various aspects of it, like computational models of emotion (FLAME, EMA, ParleE, Greta, GALAAD, etc.), behaviourbased robotics, agents systems, and many others (El-Nasr et al., 2000; Gratch and Marsella, 2004; Bui et al., 2002; Poggi et al., 2005; Levine, 2007; Bonarini et al., 2006). Still, there is a lack of projects which result in applicable models of human motivation psychology, and most of the available results are generally not suitable for our purposes. For instance, the ideas behind the acronyms EMA and FLAME are concerned with a computational model of emotions interpreted as a closed motivation-and-decision system.

It is clear that emotions are an important part of human motivation. Following this line, we propose a concept 
(Kowalczuk and Czubenko, 2010c), further built up in this work, in which system-developed emotions play the role of decision-making modifiers.

An interesting approach to implementing human emotions can be found in the work of Leite et al. (2008), where a robot expresses some emotions (apparently, robots with emotions appear even more fascinating!). Similar concepts are developed for web applications, looking for intelligent chatter-bots with emotions (Kowalczuk and Czubenko, 2010a).

Most publications on mobile robots with built-in emotions are about a direct interaction between a man and a robot, whereas we are concerned about the interaction between a robot and its environment (humans can be a part of the environment, too), which is a principal source of handling the needs.

Systems of motivation (considering needs and emotions) can be found in general psychology, and in a number of its branches. Nevertheless, in this paper we instrumentally consider the motivation system only in the context of personality psychology. It is crucial for our concept of building autonomous robots, in which we consider that motivation theory is solely a personality problem. Personality psychology dynamically investigates (discovers) differences and similarities in human behaviour. Clearly, the results and similarities obtained in this way can certainly be treated as generalizations (next) belonging to general psychology. One way or another, what we seek and what we would like to explore are needs and emotions common for human beings.

Consequently, we model human psychology for the purpose of robotic autonomous control and decisionmaking systems, by using achievements from personality psychology (motivation factors) and cognitive psychology (a framework for information processing). Our concept is based on a concrete state of emotion (crisp and fuzzy) which influences the system of needs. From a systemic point of view, emotion is treated as a dynamical state of attention, and needs along with emotions make a principal basis for inference.

\section{Basic psychology model}

Psychology is a scientific area dealing with mechanisms related to human behaviour. Among many fields of psychology, we should list a great number of basic branches of psychology: applied, behavioural, cognitive, evolutionary, gestalt, linguistic, neuro, organizational, personality, school, sensory, or social among others.

A model based on 'entire' human psychology would be too complex and incomprehensible. Therefore, in the following we shall focus our attention solely on the cognitive and personality branches of psychology. In particular, it is the theory of cognitivity which is a special basis for artificial intelligence. Important are thus the re- sults of cognitive psychology, which deals with all kinds of information processing, and relationships between memory and stimuli. It recognises how we think, and even what happens during sleep. On the other hand, personality psychology deals with the motivation to act, and with differences and similarities between individuals. Motivational factors, namely, emotions and needs, are key notions in modelling human behaviour. All this allows us to model human behaviour and ways in which human brain affects both emotions and reasons.

2.1. Cognitive psychology. In general, the cognitive approach to decision-making processes postulates that the knowledge being a basis for decisions is not simply created by passive accumulation and storage of data. Instead, active processing of data takes place. This means that the structures of human cognitive processes constitute a solid basis for modelling the decision process of thinking entities (Lindsay and Norman, 1984).

Cognitive processes can be categorized as elementary or complex, with the following elementary processes:

- sensory perception,

- discovery perception,

- unconscious attention (unintentional, or Top-Down Attention, (TDA)),

- conscious attention (intentional, or Bottom-Up Attention, (BUA)),

- memory,

and complex processes:

- thinking,

- language processes.

At this stage of the project, we do not consider any language aspects, as they actually deserve a separate mathematical/modelling treatment. Besides, in our opinion, prospective autonomous robots need a model of mind very much, whereas their language abilities can be easily constrained to a system of audio-visual messages.

Starting with stimuli appearing in receptors, information passes through perception (Nęcka et al., 2006). Next, it is filtered and processed by attention, and then analysed by thinking. Taking this into account, the resulting original and simplified model of relationships amongst cognitive processes is represented in Fig. 1.

2.1.1. Memory. Natural memory has double functionality: it exists as a process and as a collection of information. The memory process consists in strengthening memory paths, which must be done, since information, once memorized, weakens when not being used. 


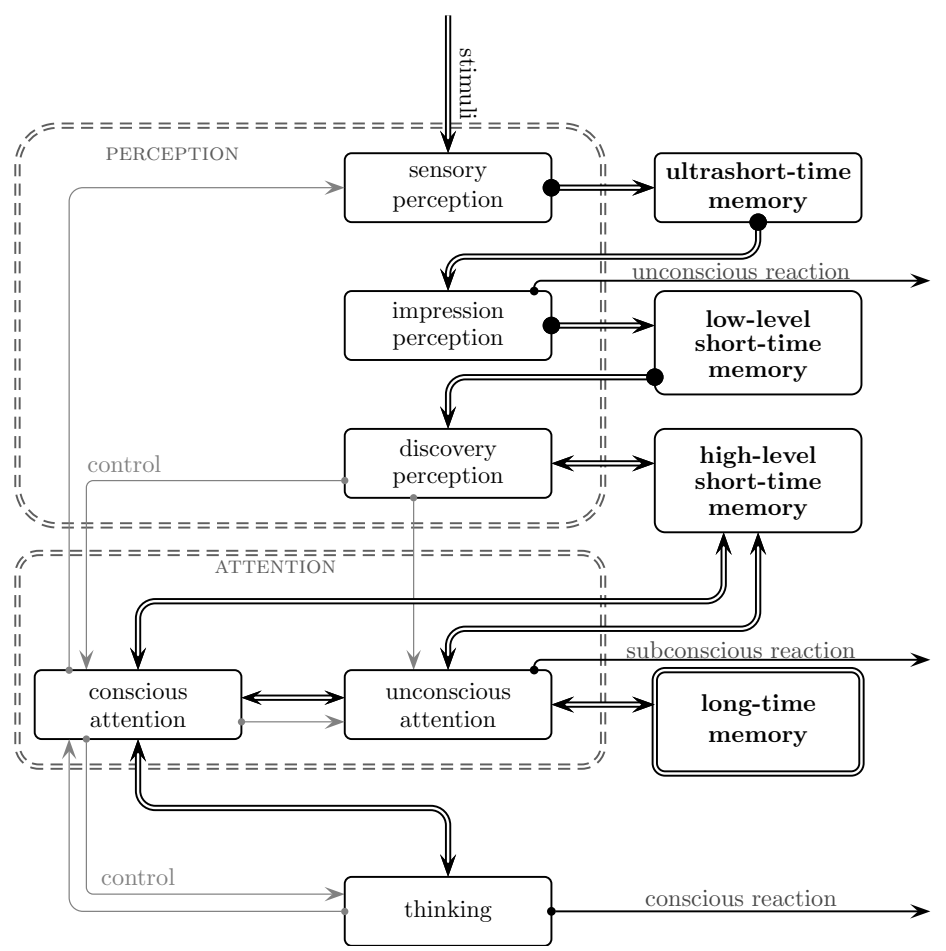

Fig. 1. Basic model of cognitive processes (along with a reaction mechanism).

On the other hand, based on the classical understanding (Maruszewski, 2001) of memory as a collection of information, we consider it a hierarchical data storage:

- sensory memory (USTM: Ultra-Short-Time Memory), used for latching and keeping stimuli (rough data or raw information);

- Short-Time Memory (STM), which is a kind of a limited container of data (abstract items needing immediate attention) that can be divided into

- low-level STM (Low-STM) keeping noticed impressions,

- high-level STM (High-STM) containing identified discoveries (objects);

- Long-Time Memory (LTM), being in line with the common sense of an abstract memory, and storing a wider set of objects and system reactions.

Note that an identified object need not to be (yet) recognised. On the other hand, some of the abstract objects of LTM convey the idea of needs, which should be recognised along with a specific impression referred to as satisfaction (level).

2.1.2. Perception. The perception system is archetypally divided into sensory and classical discovery perception. The first is in charge of rough processing of sti- muli, whereas the second one codes and recodes information, and technically is partitioned in accordance with the applied hierarchical structure of information and STM shown above, resulting in impression (low-level) and discovery (high-level) perception.

Sensory perception is responsible for the physical means of writing down raw information into raw/sensory memory (USTM). Such memory can be implemented as a simple unit built of flip-flops. At the moments of appearance of new stimuli, old information is overwritten.

Impression perception, being a preliminary stage of sensory data processing, searches and recognises simple features (like shape, color, texture, stretch, sounds, smells, touches, or other details) associated with a certain set of points in an abstract stimuli space represented by USTM. Such features are written down into impression memory (Low-STM) as impressions.

Discovery perception is a consecutive stage of sensor data processing. It consists in compiling impressions into discoveries (in a bottom-up process), which is performed allowing for space correlation (w.r.t. a locus in a meant environment). A single unnamed discovery (finding) is a group of impressions concerning a single object. Such discoveries are compared (in a top-down process) with discoveries known from discovery memory, another STM (High-STM), supplied and written down there by unconscious attention 1

\footnotetext{
${ }^{1}$ Note that discovery perception needs a bilateral communication
} 
Unrecognised discoveries make the perception send an FNO control signal (Fetch New Object) to unconscious attention. If this does not help (i.e., a given impression still does not match), a CNO control signal (Create New Object), is generated to communicate with conscious attention.

It is generally recognised (Broadbent, 1958) that, during the process of recoding impressions, perception may as well detect some dangerous features, which, consequently, can lead the entity to a specific state associated with some unconscious reactions (for example, encountering a 'mouse' can generate a sub-emotion referred to as panic).

In our interpretations of this mechanism, objects (discoveries) can have associated sub-emotions and subconscious reactions, which are part of the modelled mind process, too. They are treated here as specific impressions composing discoveries.

2.1.3. Attention. As can be seen from the developed structure (Fig. 1), attention is a core process for the whole cognition system. In general, attention has access to the short-time and long-time memories (High-STM and LTM) with discoveries (binding impressions into single objects).

Unconscious attention (TDA), based on certain control signals, updates needs, emotions, and mood. It governs memory processes (STM and LTM) and supports cognitive processes 2 In particular, it transfers memorized discoveries from LTM into High-STM. Usually, unimportant objects in STM are replaced/substituted by new ones (possibly usable). The transfer is performed upon the FNO control signal sent from discovery perception when it is unable to match impressions to any of the current objects kept in STM.

Conscious attention (BUA) is necessary to make new findings, that is, to create new objects/discoveries for sets of impressions newly identified and recognised as meaningful. This process is initiated by the CNO control signal generated by discovery perception (when all LTM solutions/objects recognised in the past have been explored and rejected). The new finding is performed (fixed) via unconscious attention by transferring a new isolated set of impressions along with its label (invented by thinking) as a new discovery to LTM. Clearly, thinking, placed at the highest level of cognition as shown in Fig. 11 is invoked by conscious attention.

Note that information always crosses the unintentional attention while being exchanged between LTM and STM. The TDA attention also filters information sent (through BUA) to the thinking process. Due to the hierarchical organization applied, only most essential pieces of information are admitted (authorized) to thinking (Maruszewski, 2001).

\footnotetext{
with its memory (High-STM).

${ }^{2}$ The next subsection on personality psychology discusses the concepts of need, emotion, and mood.
}

Reactions often repeated (in some period of time) eventually create subconscious actions (like those applied in driving cars or typing), allowing the subject to react 'without thinking', in a faster way as compared to conscious reactions. Thus, upon recognizing known memory tracks, TDA is capable of immediately generating subconscious reactions (Kahneman, 1973).

Conscious attention is also able to intentionally control both reflexes and certain cognitive processes, directly or indirectly (some results of BUA can be partly unconscious). For example, a subject can fix its attention on some impressions connected with a certain object, which improves (hones) its attention process. A known example of such a BUA feature is the so-called 'cocktail party' effect which concerns the ability of focusing attention on a sole talker in an environment full of conversations and background noise, which otherwise reflects a brain ability to strongly filter out superfluous information (Arons, 1992).

2.1.4. Thinking. Thinking is generally the most complex and developed process. It contains decision making, logical reasoning, creating plans for the future, forming concepts, and solving problems. In general, thinking can be of two categories:

- autistic thinking (also called unmeant thinking), responsible for all kinds of imaginary processes, dreams, brain-in-resting, and excrescent thoughts (Dewey, 1997);

- realistic thinking, solving problems by (simple, stepby-step) logic operations relevant to the theories of sets and models, as well as to constructive mathematics and other fields of logic (Monsell, 1996).

2.1.5. Language $3^{3}$. Language processes are responsible for making articulated sounds, forming sentences, etc. A vivid dispute between behaviourists and their opponents in the beginning of the history of cognitive psychology concerned the language processes. Today, it is generally accepted that the processes are strongly associated with memory, as well as that certain rules are coded by a respective strand of nucleotides (DNA and RNA) (Maruszewski, 2001).

2.1.6. Model of cognitive psychology. As has been presented in Fig. 1] cognitive processes can be arranged in a hierarchy. Indeed, this is an extremely simplified model, as a high-fidelity one would need a complex neural network of an enormous size. It is estimated that human brain contains $10^{11}$ neural cells, and every neural cell has about 7000 neural connections. Apparently, even after eliminating the challenging uncertainty existing in

\footnotetext{
${ }^{3}$ In this work the language aspects are considered to be integrated within functions of thinking, and will not be discussed separately.
} 
our knowledge about the (human) brain, there are no computers which could practically handle such a kind of web (Drachman, 2005).

Clearly, there are some control signals necessary for directly coupling particular cognitive processes. In such a way, a thinking entity is able, for instance, to pay its attention to one of the discoveries made by the perception process. It means that the attention process can ordain a deeper analysis of an object under consideration.

2.2. Modelling motivation factors. Psychology which takes care of individual differences is referred to as personality psychology. This psychology is, in particular, responsible for both creating a coherent model of a person and tracking individual human differences. Moreover, it studies human nature, motivations and emotions. The key element, from the viewpoint of autonomy of robots, is the nature of motivations and emotions.

Consider thus the two kinds of motivation factors: needs and emotions, which are the most important quantities for our analysis. Needs are principal elements of human motivation. It is only in exceptional cases, when a thinking entity has to react immediately, that its system of reaction (motivation/needs) does not directly engage its reason. In such moments, reaction simply corresponds to emotions. As will be shown below, emotions are also input (controlling) data for the system of needs.

\subsubsection{Modelling personality psychology by needs.} Generally, a need is an abstract state of an entity experiencing a sense of dissatisfaction (Maslow, 1968). The stronger the sense, the harder it should be eliminated (or, at least, reduced). There are a huge number of needs, which can be divided into several classes shown in Fig. 2, along with some sample items (needs).

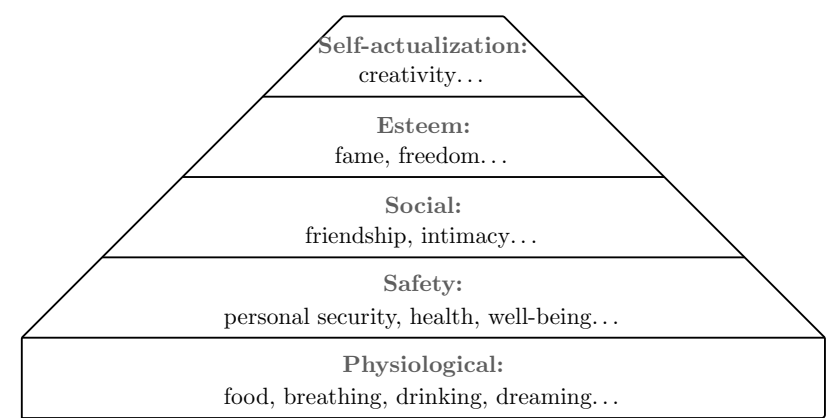

Fig. 2. Maslow pyramid of needs.

Children have about 26 needs, spread within all the Maslow classes. In human lifetime a number of new needs are hammered out. The number of needs has no special significance for our development (it is only an implementation issue). Needs can be defined by the designer, an can be self-created, according to the emergence of new tasks to be performed by the agent, for instance.

The perspective of building autonomous robots implies the existence of certain extra 'needs' connected with their subordinate tasks (within an assumed hierarchy), as compared to the entities created based solely on the human prototype system of needs. Soldiers obeying duty orders constitute a practical archetype at this point. Such orders can be directly related to the safety needs and (to some extend) to the 'social/belonging' needs. Nevertheless, for the sake of simplicity, we assume that such external subordinate tasks are existential, and thus adhere to the category of safety.

2.2.2. Emotional process. Emotional processes can have different forms (Biddle et al., 2000):

- sub-emotions, represented by rapid passing through feelings related to a recognised object;

- classical emotions, denoting changeable affective states, with a strong color and explicit valuation;

- a mood, having a weaker color and longer duration time (Blechman, 1990).

Leaving aside various deliberations or speculations on creating emotions (mainly due to their involved and equivocal nature), we claim that emotional processes with respect to an object can vary between strong negative and positive feelings connected to both sensed stimuli and changing extends of fulfilments of needs.

Emotional processes fulfil four functions: information oriented, activating, meta-cognitive, and modulating. Their main utility in the developed psychology model is modulation, which is responsible for extending or restricting the sense of fulfilment of needs. The informative function simply deepens (adds more) information about a perceived object, and thus facilitates its qualification, while the other two functions of emotion help getting more information about this object (Ekman and Davidson, 1999) by a direct influence on the current process of cognition (the meta-cognitive function switches the method of cognition/creation, and activation finds a new viewpoint/discovery in LTM).

From a linguistic standpoint, one can distinguish over thirty emotions. Most of them, however, are strictly related (linearly dependent), thus they will not be quoted here (Plutchik, 2001)

The employed simplified interrelations between needs and emotions depicted in Fig. 3 represent our interpretation of the natural idea of emotions. The division of emotions commonly used in psychology (Biddle, 2000) concerns emotions and moods, as well as affects. Our sub-emotions should be associated with affects, which rely on certain memorized objects. As the classical emo- 
tions are influenced by sub-emotions, they are also (indirectly) connected to agent system memory: As different attributes can be associated to any recognised object (discovery) kept in memory, among them, there can be memories of experienced emotions (impressions) associated with such a memorized object. In view of the above, our sub-emotion means simply a kind of signal, which comes from system memory.

Consequently, needs are verified by unconscious attention (TDA) according to the recognised discoveries, as some of them represent the robot's needs. The instrumental satisfaction level of a need is thus (also) modelled as a specific impression held in a respective discovery. Furthermore, TDA is responsible for transforming the actual states of all agent needs into a scalar image of the classical emotions, which can be modulated by sub-emotions, too. Mood is generated according to the evolution of this emotion image (Figs. 5 and 6 .

The general idea of the implemented mechanism of mood explained in Fig. 6refers to the system's dynamics (associated with the changeable classical emotion), along with the employed restricted range of states of emotion. Note that mood is going down to its lowest value (' -1 ') in the case of negative emotions (exactly the negative values of the classical emotion), according to a slanted hysteresis shown in Fig. 6. Actually, the emotion mechanism also depends on time (this effect 4 is now only partially and indirectly taken into account, for instance, in a transient/temporary influence of sub-emotions). Note that 'anger' is in our case only a label of a 'lowest' emotion. For example, after getting a sentence, the mood of a wrongdoer is going down according to (a change of) his negative emotions (especially, for a certain period of time), and such an extremely negative state of emotion (for the judge and the whole world) is marked as 'anger'.

The mood influences the system of needs. A general concept of changing the satisfaction limits is based on the system of self-awarding applied. When the agent's behaviour is profitable and well rated, it is prized. We apply a specific award in the form of 'relaxation', which consists in broadening the satisfaction limits (consequently, such relaxation can be interpreted as being 'free' from anxiety, and thus 'easy going').

\section{IDS: Intelligent decision-making system and its integral model}

The basic model of our Intelligent Decision-making System (IDS) shown in Fig. 1 needs further refinements and definitions, especially with respect to the ways of using the needs. According to the literature (Nęcka et al., 2006) the needs take shape right after a preliminary selection of perceptions, de facto in the time moments determined by

\footnotetext{
${ }^{4}$ It certainly calls for further studies.
}

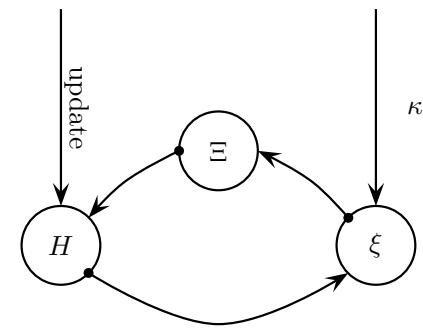

Fig. 3. Modelling personality psychology as a relation between sub-emotions $(\kappa)$, classical emotion $(\xi), \operatorname{mood}(\Xi)$ and needs $(H)$.

attention. The needs, in turn, exert their influence on the decision process and, consequently, on the system reaction.

3.1. Modelling the importance of needs. A static importance of the needs can be partly determined according to the pyramid of Fig. 2. Obviously, physiological needs are more important than those of safety, which, in turn, are prior to the socially belonging needs, etc.

This is, however, not all, especially from the problem sorting/weighing point of view. Clearly, needs should also be dynamically differentiated in terms of their importance within each class (on one pyramid level). The dynamic importance of the needs can be related to a concrete degree of their fulfilment.

Fuzzy set methodology will be used here for this purpose, as well as for modelling and processing reasons. Let us first assume that each need can be quantified in terms of fulfilment based on three fuzzy sets attributed to it. Their membership functions are determined by certain rudimentary parameters describing this need. Consequently, it results that each need can be in a state of satisfaction (appeasement), pre-alarm, or alarm. It is clear that the latter absolutely requires some reaction.

Thus, taking into account such an alarming property, the following weighting function is proposed 5 :

$$
\omega\left(\eta_{i} ; c\right)=\frac{1}{1+\exp \left(-f_{s c}(c)\left(\left|\eta_{i}\right|-c\right)\right)},
$$

where $\eta_{i}$ represents an actual value of the degree of fulfilment of a given $i$-th need, and the coefficient $c$ is a mean value of the membership parameters describing the fuzzy sets of satisfaction and alarms. For a proper representation of the weighting (thresholding) function and its inflection point, the scaling function has been selected to have the following form:

$$
f_{s c}(c)=0.1+0.00025(c-50)^{2} .
$$

\footnotetext{
${ }^{5}$ A sigmoidal function.
} 


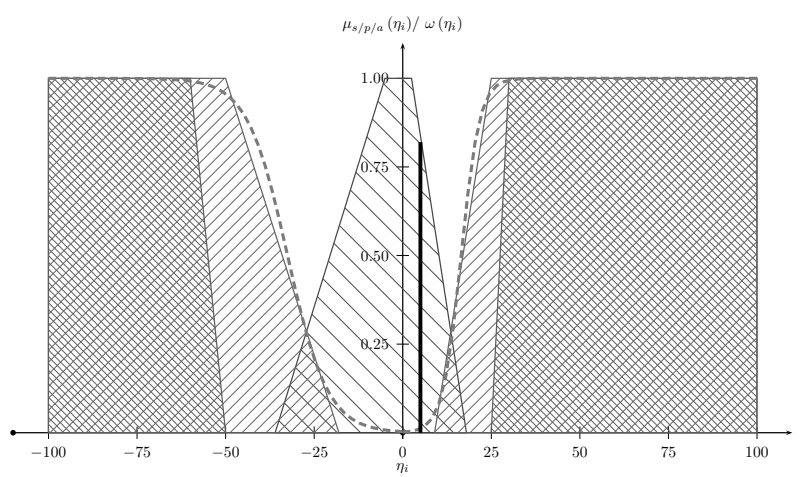

Fig. 4. Sample fuzzy membership of an $i$-th need and its estimates. The bold dashed line denotes the weighting function $\left(\omega\left(\eta_{i}\right)\right)$, the sparsely hatched-backslashed area describes the satisfaction set $\left(\mu_{s}\left(\eta_{i}\right)\right)$, the densely hatchedcrossed area portrays the alarm set $\left(\mu_{a}\left(\eta_{i}\right)\right)$, and the densely hatched-slashed area means the pre-alarm set $\left(\mu_{p}\left(\eta_{i}\right)\right)$. The thick vertical line marks an actual value of the fulfilment degree $\left(\eta_{i}\right)$.

A sample fuzzy classification of a need is shown in Fig. 4. According to this figure, it is clear that in its moments of satisfaction the need is not important, whereas in the alarm cases the need gets its highest ranks (weights).

3.2. Modelling the emotions. The emotions are some states of mind, which modify the system of needs and reactions. They deliver a simple mechanism of protection from danger, and give rise for awarding the agent. For example, when the emotion of fear is invoked, an intelligent robot can be programmed to immediately run away from danger, instead of continuing with its basic duty/tasks (like weeding a garden).

Sub-emotions can be interpreted as 'impressions' connected with some objects (like a pink blanket from childhood, or a beloved woman, for instance). In our concept, sub-emotions are additional qualifications of discoveries, other than the sensory impressions founding them.

Let us consider, for simplicity, that the classical emotion is reduced to a single variable, and that sub-emotions are trivalent negative, neutral, positive. In such a way, a current sub-emotion (of a recognised object) can incrementally modulate the classical emotion (see Fig. 3).

A normalized domain of the classical emotion is decomposed into seven fuzzy sets. Each of the sets represent a single fuzzy emotion. In such a way we can linguistically differentiate between fear, anger, sadness, indifference, happiness, curiosity and joy. A complete set of all respective membership functions is depicted in Fig. 5. The selected set of fuzzy emotions can be considered a certain confinement of Plutchik's theory (Plutchik, 2001). The employed mechanism of emotions (again a modification of Plutchik) makes allowances for a convenient optimization/selection of reactions to be taken.

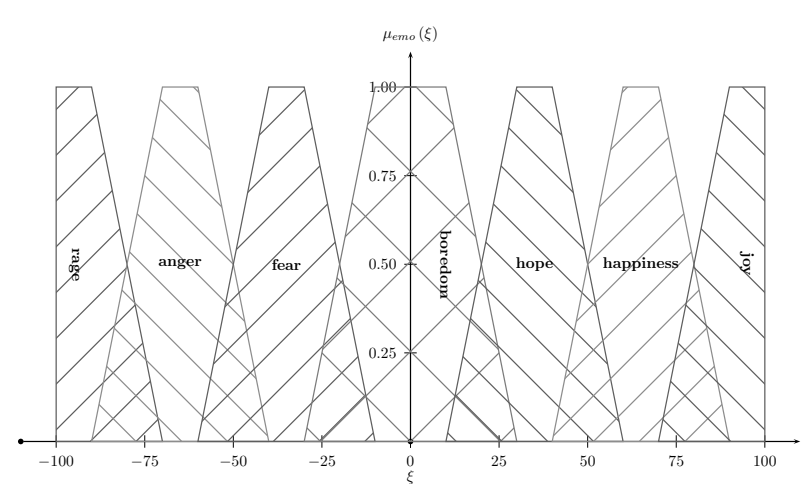

Fig. 5. Fuzzy model of classical emotions $(\xi)$. Instantaneous values of the emotion are on the $\mathrm{X}$-axis $(\xi)$, the membership functions represent (from the left to the right) respectively rage, anger, fear, boredom, hope, happiness, and joy.

The third occurrence of the emotional processes is the mood (Fig. 6). Its variable is formed according to the evolution of the classical emotion submitted to the slanted hysteresis operation applied. The mood moderates the fuzzy membership parameters of the needs.

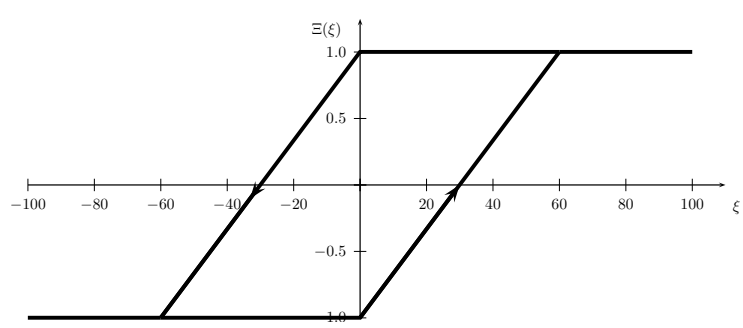

Fig. 6. Dependence between the mood and the classical emotion states.

3.3. Integral IDS model. An integral model of cognitive processes, shown in Fig. 77 has been built by assimilating the developed personality psychology elements model into the basic cognitive model. Parts of personality have arisen from the discussed models of conscious and unconscious attentions. Note that in this figure the sensory perception are merged with the other perceptions and two levels of memory (USTM and Low-STM).

Abstract and reaction memories have been isolated within LTM. The abstract memory contains all known discoveries categorized and systematized in groups of more generalized discoveries (for example, chair, sofa, coach, stool, seat, and pouf, can be generalized as seat-places). The reaction memory, on the other hand, contains all po- 




Fig. 7. Integral IDS model of cognitive processes from the viewpoint of personality psychology.

ssible reactions (with their parameters) that can be utilized by the system/agent.

3.4. IDS model vs. decision theory. There exists a specific field of science, called decision theory, that concerns the problems of forming and solving decision tasks (Hansson, 1994). One of most sophisticated classical models of decision making, referred to as the Mintzberg model, is shown in Fig. 8 .

The studies of Mintzberg et al. (1976) prove that the most time-consuming element of the model is identification. Note that in our concept this function is automatically performed by the whole system centred around needs.

Clearly, finding a known solution appears to be the simplest and fastest method. In this respect, the Mintzberg paradigm is answered by our integral model (which otherwise involves an explicit 'thinking' process). With a great degree of similarity, in both cases we can distinguish the same phases: perception (getting information), attention (identification of a problem), and thinking (resolving the problem).

3.5. IDS thinking process. The principal element of cognitive processes is thinking, which can be divided into autistic and realistic. Autistic thinking is subconscious; in our model there are thus some artifacts of it. Realistic thinking completes three basic functions (Nęcka et al., 2006):

- creative: improves known reactions and creates new reactions (in a genetic way, for instance);
- re-constructive: chooses a currently most well-fitting reaction;

- estimation: creates an estimation for reactions applied, as well as classifies them into unconscious and subconscious reactions.

Needs and emotions are signals that influence both creative and re-constructive thinking as shown in Fig. 9 They make a principal basis for undertaking proper reactions. In a creative-thinking process of the IDS, a new reaction is originated that should 'satisfy' its system of needs to a possibly greatest extent. As an effect, resulting amendments (increments) of the employed satisfaction measures of all the needs obtained by this (being experienced) reaction are memorized, along with the context of current emotions (namely, one or two; see Fig. 5). As will be shown in the next subsection, in re-constructive thinking the IDS can consider most fitted reactions in another current context of emotion.

Consequently, emotions modify the pool of accessible reactions. For instance, a reaction in a form of 'fight' should be applied only in case of danger. When environment is malevolent, the emotion system generates and adapts signals of negative sub-emotion (connected with some recognised objects of an unfriendly environment). In a next step, attention (associated with the personality subsystem depicted in Figs. 3 and 7) modifies/decrements the classical/system emotion, leading to its negative crisp value (see Fig. 5) and the negative fuzzy emotions (fear, anger, or rage). This, in turn, lets the agent react violently (e.g., by employing power or fighting). 

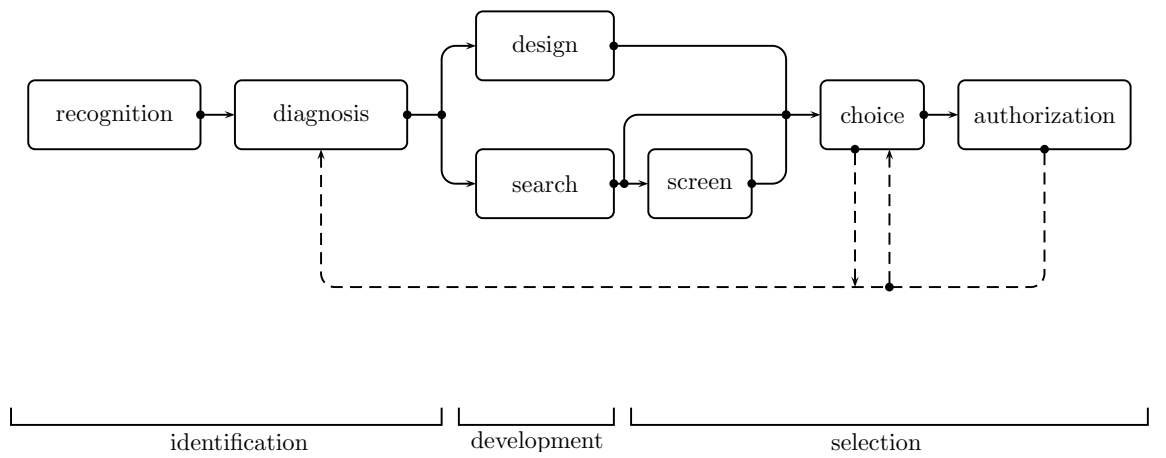

Fig. 8. Classical model of the decision-making process (Hansson, 1994).



Fig. 9. IDS thinking module.

3.6. Decision making mechanism: Motivations. Within the frameworks of thinking, one straightforward solution implemented at this stage of our study takes into account only a re-constructive model, where all possible reactions are assumed to be known and the only task is to select a suitable reaction according to a current state of needs and emotions. Even such an apparently simple task can get easily complicated 6 when a part of knowledge is 'crisp' and one has to consider several criteria or needs.

To give some rationale for the proposed solution, let us analyse the issue of complexity. As has been mentioned, an average infant has about $n=26$ different needs, and about $r=20$ complex reactions (mostly moves and sounds). Let us think about building a simple version of a robotic system inspired by the employed anthropoidal model that fulfils these assumptions.

Let us thus approximately compare two sample distinct approaches to the decision making problem: CDM,

\footnotetext{
${ }^{6}$ Giving rise to another encounter of the 'curse of dimensionality.'
}

optional Crisp with Data Mining (associating a fixed decision for each condition), and FNN, Fuzzy with Neural Network (referred to as a fuzzy-neural network estimating/weighing/considering a possible effect of applying each of available reactions, and presented in the next subsection).

Assuming 8-bit representation of the state of needs and the set of 26 needs, the crisp approach (in view of the choice of a single reaction) yields the number of necessary decisions equal to the number of possible variations: $256^{26} \cong 4.1 \mathrm{e} 62$. When we have only 7 needs (as in the robot experiment described in Section 5), the database would have about 7.2e16 records, whereas in the case of FNN we have only $3 n r$ data to be input to the decision network, which is 1260 for the infant case (and 105 for the robot case).

Certainly, the proposed FNN solution has its own complexity, resulting from the structure of neurons 'AND' (3), (5), 'OR' (4), (6), and 'NOT' (7), applied later on. As a consequence, we can estimate the FNN complexity in terms of 3300 additions and 1660 multiplications (and, for the robotic experiment, 255 additions and 130 multiplications).

Thus, considering the above indicated great number of possibilities, the decision optimization problem can be simplified and conveniently solved by FNN simulating the effect of reaction, instead of keeping a huge set of circumstances and reactions in extenso in the agent memory (CDM). Additional advantage of the FNN approach can be found in its adaptivity to possibly changing decisionmaking conditions.

3.7. Selecting reactions with the use of a fuzzy-neural network. The decision on the reaction to be undertaken is made by optimizing a combined weighted criterion composed of a (maximized) satisfaction level and (minimized) a distress level, with respect to the alarm and prealarm fuzzy thresholds.

Therefore, based on a priori information about in- 


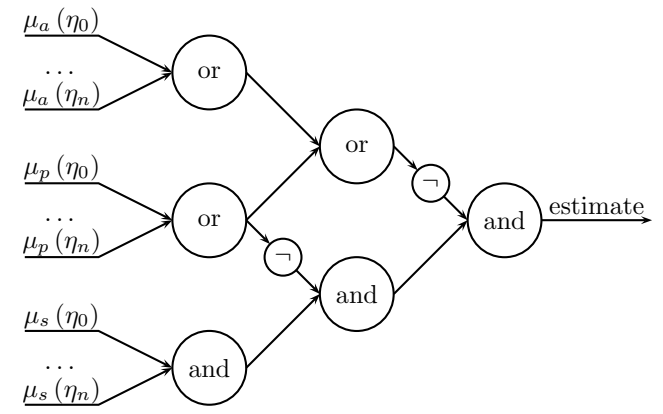

Fig. 10. Fuzzy-neural network estimating the suitability of reactions based on the actual state of needs and the simulated effects of reactions.

cremental effects of the reactions on the needs, for each reaction, a 'simulation run' is performed in order to estimate its influence on the system of needs. The obtained new fulfilment degrees and the importance weights of all the simulated needs are then taken into account as the input information applied to the neural network of Fig. 10.

The first (lower) neuron reflects fuzzy operations between the membership of the needs to the fuzzy satisfaction set and the respective weights of the needs. The second neuron considers the fuzzy pre-alarm set, and the third neuron computes the fuzzy alarm set (similarly, both use the fuzzy membership and the weightings).

The employed neuron of type 'AND' can be described by the following function:

$$
y=f_{\text {and }}(\mathbf{u})=\bigotimes_{i=1}^{N}\left(w_{i} \circ u_{i}\right),
$$

where $\otimes$ denotes an $N$-argument T-norm, and o represent a two-argument $\mathrm{S}$-norm.

The neuron of type 'OR' is computed as follows:

$$
y=f_{\text {or }}(\mathbf{u})=\bigcirc_{i=1}^{N}\left(w_{i} \otimes u_{i}\right),
$$

where $\bigcirc$ denotes an $N$-argument S-norm, and $\otimes$ is a twoargument T-norm.

Both the T-norm and the S-norm are assumed to be in the form of the following Einstein norms (Łęski, 2008):

$$
\begin{gathered}
\otimes_{E}(x, y)=\frac{x y}{2-(x+y-x y)}, \\
\bigcirc_{E}(x, y)=\frac{x+y}{(1+x y)} .
\end{gathered}
$$

The symbol $\neg$ represents negation in the Yager sense:

$$
N(x ; s)=\left(1-x^{s}\right)^{\frac{1}{s}} .
$$

In the proposed fuzzy-neural network, estimating the effect of reactions, the negation parameter $s=2$ is taken on as a simple optional choice.
The network generates its estimates as real values from the interval $[0,1]$, which results in a convenient ordering of reactions. The best reaction is forwarded for execution, with the expectation that the system states of needs will be improved.

The FNN input data are fuzzy images of the fulfilment, where fuzziness concerns alarming, pre-alarming, and satisfaction sets, according to Fig. 4.

The first layer of the fuzzy-neural network represents a data collecting operation. The satisfaction neuron ('AND') assumes that each need should be satisfied; the output of this neuron is an estimate of global satisfaction (a low value of the estimate shows that more needs remain unsatisfied). The 'OR' neurons for pre-alarms and alarms take care of keeping all the needs away from being (pre)alarmed.

The second layer of FNN computes co-estimates: a Satisfaction Pre-alarm co-Estimate (SPcE) and an AlarmPre-alarm co-Estimate (APcE). The SPcE can be interpreted as follows: 'Every need should be satisfied and none of them should be pre-alarmed'. On the other hand, AP$\mathrm{cE}$ shows an estimation of dissatisfaction. The last layer creates a single evaluation of the state of system needs.

In conclusion, the result of each reaction is simply computed by performing a simulated estimation (called 'simulation') of the effects of its application. This estimation is performed by the FNN and based on data representing the state of fulfilment of the $(n)$ needs considered. The respective vector of the need fulfilment states is vital to the IDS. Note that, actually, the 'needs' shown in Fig. 7 are technically implemented as a bus of lines, each of which represents the current (crisp) state of fulfilment of an ascribed need.

Known reactions are memorized in LTM along with their learned incremental influences (amendments) on particular needs. Thus, in view of the above, the inferencing machine is able to foresee the 'environmental response' (distributed over the whole set of needs), and, in effect, to estimate the effect of any agent reaction, and to select the best one (that is, to optimize the decision).

\section{Fuzzy-neural network experiments}

Let us assume that the IDS recognises the following needs, which are accordingly associated with the respective Maslow classes (levels):

- En: Energy (Physiological),

- Re: Rest (Physiological),

- Se: Security (Safety),

- Ta: Tasks (Safety),

- So: Society (Social), 
- Cu: Curiosity (Esteem),

- De: Development (Self-actualization).

All the needs have their specific fuzzy parameters defined, which precisely describe their membership functions. Moreover, it is assumed that this IDS has five predesigned reactions:

- Searching (area),

- Charging,

- Resting,

- Working,

- Escaping.

An FNN module of a Java-based framework, relying on JAMA and FuzzyJ Toolkit libraries, was created and used in simulation. The effects of the IDS decisionmaking tests performed with the aid of the FNN module are described and discussed below. The influence of particular reactions on the state (degree) of fulfilment of the assumed needs is known and estimated as described in Table 1.

Computational experiments, based on the above described fuzzy-neural network, were conducted with the purpose of searching for the best-fitted reactions in three sample scenarios, called experiments E1-E3. Table 2 presents the resulting estimated transitions (see the rows) in the states of fulfilment of the needs (initially described in the first aggregate column), and in terms of the prospective crisp (FD) and fuzzy (LS: (s) satisfaction, (p) pre-alarm, and (a) alarm) states of the needs, gained by the five reactions considered (the other double columns).

As can be seen, initially, there is only one need in the alarm state in the scenario E1. Consequently, we look for a reaction that most optimally influences this need. The obtained values of the estimated suitability are given in Table 3. Clearly, in the case E1, with the entity feeling insecure, escaping appears to be most optimal.

The second experiment (E2) shows what happens when there are two unsatisfied needs (Energy and Task). As Table 3 shows, the IDS advises a reaction ensuring its survival, i.e., it prefers to gain energy (charging) instead of performing the assigned task (this specific need will stay alarmed, as Table 2 prompts), programmed as working (e.g., painting).

There are four alarms in the last scenario (E3). In such situations it is especially helpful that the estimated suitability depends on the fuzzy parameters of the needs. The IDS has always a chance to choose a reaction which is most suitable.

The presented simulation proves that the FNN system is able to choose a single reaction capable of handling the system of needs. The employed scalar function of the FNN allows on-line solving of a difficult multioptimization problem appearing here (note that fulfilling each need represents a different criterion).

\section{Robotic implementation and experiments}

As has been mentioned, the principal idea of this paper is focused on the concept of needs (with an auxiliary and instrumental role of emotions). At this stage of study, we can also present results of a simplified experiment based solely on the implemented concept of needs (which, certainly, is partial when taking into account a complete IDS viewpoint).

The resulting simplified version of the IDS was implemented on a laboratory mobile platform Qfix, which has four distance sensors, four diodes and two electric motors. The reactivity of the robot was quite limited, since it could only move in two dimensions, sense objects in its closest environment, and change the states of four diodes.

5.1. Robots needs. Due to the above mentioned limitations, we assume that the robot has four needs:

- eating (intent to have high energy in its battery),

- resting (desire to stay at a current location),

- safety (aim to find a shelter),

- curiosity (desire to move to new places).

The eating and resting needs are in the physiological class, and the safety and curiosity needs are in the safety class of the Maslow pyramid (no emotions and mood were considered in this simplified experiment).

5.2. Implementation report. Let us assume that one reaction positively influences (corresponds to) each need. Thus, there are the following reactions:

1. 'charge' (charging the battery is indicated by a blinking diode),

2. 'hide' makes the robot go to a nearest corner,

3. 'sleep' results in resting,

4. 'explore' lets the robot go beyond known positions (marked on its map).

All performed movements (reactions) result in loosing energy and changing the states of satisfaction of other needs (which are also decreasing in time).

To facilitate observations, the robot needs and states were indicated by diodes mounted on the platform. In general, the robot acted as a 'baby', according to the above given description of the implemented 'program' of satisfying the robot needs: by running to a corner, charging, 
Table 1. Examined reactions and their simulated effect on the state of fulfilment of particular needs.

\begin{tabular}{|c|c|c|c|c|c|c|c|}
\hline Reaction & Energy & Rest & Security & Tasks & Society & Curiosity & Development \\
\hline \hline Searching & -20 & -20 & -15 & - & - & +30 & +25 \\
\hline Charging & +40 & +10 & -25 & -5 & +5 & -10 & - \\
\hline Resting & +10 & +30 & -5 & - & - & - & +5 \\
\hline Working & -30 & - & - & +30 & - & - & - \\
\hline Escaping & - & -20 & +30 & - & -10 & - & - \\
\hline
\end{tabular}

Table 2. Sample estimation of reactions gained by the fuzzy-neural network in terms of the obtained fulfilment of the needs, where LS means the linguistic state (s: satisfaction, p: pre-alarm, a: alarm), FD is the degree of fulfilment.

\begin{tabular}{|c|c|c|c|c|c|c|c|c|c|c|c|c|c|}
\hline ID & \multicolumn{3}{|c|}{ Needs } & \multicolumn{2}{|c|}{ Searching } & \multicolumn{2}{|c|}{ Charging } & \multicolumn{2}{|c|}{ Resting } & \multicolumn{2}{|c|}{ Working } & \multicolumn{2}{|c|}{ Escaping } \\
\hline \multirow{8}{*}{ E1 } & Need & $\overline{\text { LS }}$ & $\overline{\text { FD }}$ & $\overline{\text { LS }}$ & $\overline{~ F D}$ & $\mathbf{L S}$ & $\overline{\text { FD }}$ & $\overline{\text { LS }}$ & $\overline{\text { FD }}$ & $\overline{\mathbf{L S}}$ & $\overline{\text { FD }}$ & $\overline{\text { LS }}$ & $\overline{\overline{F D}}$ \\
\hline & En & $\mathrm{s}$ & 0.0 & $\mathrm{~s}$ & $\begin{array}{l}-20.0 \\
\end{array}$ & $\mathrm{p}$ & 40.0 & $\mathrm{~s}$ & 10.0 & $\mathrm{~s}$ & $\begin{array}{l}-30.0 \\
\end{array}$ & $\mathrm{~s}$ & 0.0 \\
\hline & $\operatorname{Re}$ & $\mathrm{s}$ & 0.0 & $\mathrm{~s}$ & -20.0 & $\mathrm{~s}$ & 10.0 & $\mathrm{~s}$ & 30.0 & $\mathrm{~s}$ & 0.0 & $\mathrm{~s}$ & $\begin{array}{l}-20.0 \\
\end{array}$ \\
\hline & $\mathrm{Se}$ & $\mathrm{a}$ & -75.70 & $\mathrm{a}$ & -90.70 & $\mathrm{a}$ & -100.0 & $\mathrm{a}$ & -80.70 & $\mathrm{a}$ & -75.70 & $\mathrm{p}$ & -45.70 \\
\hline & $\mathrm{Ta}$ & $\mathrm{s}$ & 0.0 & $\mathrm{~s}$ & 0.0 & $\mathrm{~s}$ & $\begin{array}{c}-5.0 \\
\end{array}$ & $\mathrm{~s}$ & 0.0 & $p$ & 30.0 & $\overline{\mathrm{s}}$ & 0.0 \\
\hline & So & $\mathrm{s}$ & 0.0 & $\mathrm{~s}$ & 0.0 & $\mathrm{~s}$ & 5.0 & $\mathrm{~s}$ & 0.0 & $\mathrm{~s}$ & 0.0 & $\mathrm{~s}$ & $\begin{array}{l}-10.0 \\
\end{array}$ \\
\hline & $\overline{\mathrm{Cu}}$ & $\mathrm{s}$ & 0.0 & $\bar{p}$ & 30.0 & $\mathrm{~s}$ & -10.0 & $\mathrm{~s}$ & 0.0 & $\mathrm{~s}$ & 0.0 & $\mathrm{~s}$ & 0.0 \\
\hline & $\overline{\mathrm{De}}$ & $\mathrm{s}$ & 0.0 & $\mathrm{~s}$ & 25.0 & $\mathrm{~s}$ & 0.0 & $\mathrm{~s}$ & 5.0 & $\mathrm{~s}$ & 0.0 & $\mathrm{~s}$ & 0.0 \\
\hline \multirow{8}{*}{ E2 } & Need & LS & FD & $\mathbf{L S}$ & FD & $\mathbf{L S}$ & FD & LS & FD & $\mathbf{L S}$ & FD & $\mathbf{L S}$ & $\overline{\text { FD }}$ \\
\hline & En & $\mathrm{a}$ & -76.55 & $\mathrm{a}$ & -96.55 & $\mathrm{p}$ & -36.55 & $\mathrm{p}$ & -66.55 & $\mathrm{a}$ & -100.0 & $\mathrm{a}$ & -76.55 \\
\hline & $\operatorname{Re}$ & $\mathrm{s}$ & 0.0 & $\mathrm{~s}$ & -20.0 & $\mathrm{~s}$ & 10.0 & $\mathrm{p}$ & 30.0 & $\mathrm{~s}$ & 0.0 & $\mathrm{~s}$ & -20.0 \\
\hline & $\mathrm{Se}$ & $\mathrm{s}$ & 0.0 & $\mathrm{~s}$ & -15.0 & $\mathrm{~s}$ & -25.0 & $\mathrm{~s}$ & -5.0 & $\mathrm{~s}$ & $\overline{0.0}$ & $\mathrm{p}$ & 30.0 \\
\hline & $\mathrm{Ta}$ & $\mathrm{p}$ & -83.40 & $\mathrm{p}$ & -83.40 & $\bar{a}$ & -88.40 & $\mathrm{p}$ & -83.40 & $\mathrm{p}$ & -53.40 & $\mathrm{p}$ & -83.40 \\
\hline & So & $\mathrm{s}$ & 0.0 & $\mathrm{~s}$ & 0.0 & $\mathrm{~s}$ & 5.0 & $\mathrm{~s}$ & 0.0 & $\mathrm{~s}$ & 0.0 & $\mathrm{~s}$ & -10.0 \\
\hline & $\mathrm{Cu}$ & $\mathrm{s}$ & 0.0 & $p$ & 30.0 & $\mathrm{~s}$ & $\begin{array}{l}-10.0 \\
\end{array}$ & $\mathrm{~s}$ & 0.0 & $\mathrm{~s}$ & 0.0 & $\mathrm{~s}$ & 0.0 \\
\hline & $\overline{\mathrm{De}}$ & $\mathrm{s}$ & 0.0 & $\mathrm{p}$ & 25.0 & $\mathrm{~s}$ & 0.0 & $\mathrm{~s}$ & 5.0 & & 0.0 & $\mathrm{~s}$ & 0.0 \\
\hline \multirow{8}{*}{$\mathbf{E 3}$} & Need & $\mathbf{L S}$ & $\overline{\text { FD }}$ & $\mathbf{L S}$ & FD & $\mathbf{L S}$ & FD & $\mathbf{L S}$ & FD & $\overline{\mathbf{L S}}$ & $\overline{\text { FD }}$ & $\mathbf{L S}$ & $\overline{\text { FD }}$ \\
\hline & En & $\bar{a}$ & -72.35 & $\mathrm{a}$ & -92.35 & $\mathrm{~s}$ & -32.35 & $\mathrm{p}$ & -62.35 & $\bar{a}$ & -100.0 & $\mathrm{a}$ & -72.35 \\
\hline & $\mathrm{Re}$ & $\mathrm{a}$ & -81.07 & $\bar{a}$ & -100.0 & $\mathrm{p}$ & -71.07 & $\mathrm{~s}$ & -51.07 & $\mathrm{p}$ & -81.07 & $\bar{a}$ & -100.0 \\
\hline & $\mathrm{Se}$ & $\mathrm{a}$ & -75.32 & $\mathrm{a}$ & -90.32 & $\mathrm{a}$ & -100.0 & $\mathrm{a}$ & -80.32 & $\mathrm{p}$ & -75.32 & $\mathrm{p}$ & -45.32 \\
\hline & $\mathrm{Ta}$ & $\mathrm{a}$ & -81.11 & $\mathrm{p}$ & -81.11 & $\bar{a}$ & -86.11 & $\mathrm{p}$ & -81.11 & $\mathrm{p}$ & -51.11 & $\mathrm{p}$ & -81.11 \\
\hline & So & $\mathrm{s}$ & 0.0 & $\mathrm{~s}$ & 0.0 & $\mathrm{~s}$ & 5.0 & $\mathrm{~s}$ & 0.0 & $\mathrm{~s}$ & 0.0 & $\mathrm{~s}$ & -10.0 \\
\hline & $\mathrm{Cu}$ & $\mathrm{s}$ & 0.0 & $\mathrm{p}$ & 30.0 & $\mathrm{~s}$ & -10.0 & $\mathrm{~S}$ & $\overline{0.0}$ & $\mathrm{~S}$ & 0.0 & $\mathrm{~S}$ & $\overline{0.0}$ \\
\hline & $\overline{\mathrm{De}}$ & $\mathrm{s}$ & 0.0 & $p$ & 25.0 & $\mathrm{~s}$ & 0.0 & $\mathrm{~s}$ & 5.0 & $\mathrm{~s}$ & 0.0 & $\mathrm{~s}$ & 0.0 \\
\hline
\end{tabular}

resting, etc., thus 'existing' for its own purposes. The next intended step is to construct an extended implementation of the developed anthropoidal model of psychology.

Table 3. Reactions and their Estimated Suitability (ES) in the three experiments.

\begin{tabular}{|c||c|c|c|}
\hline Reaction & $E S_{E 1}$ & $E S_{E 2}$ & $E S_{E 3}$ \\
\hline \hline Searching & 0.357 & 0.668 & 0.695 \\
\hline Charging & 0.356 & $\mathbf{0 . 8 5 0}$ & $\mathbf{0 . 8 0 0}$ \\
\hline Resting & 0.364 & 0.676 & 0.798 \\
\hline Working & 0.410 & 0.727 & 0.744 \\
\hline Escaping & $\mathbf{0 . 9 7 3}$ & 0.668 & 0.769 \\
\hline
\end{tabular}

\section{Conclusions}

In this paper we have contemplated the idea of creating a fully autonomous robotic decision-making system, able to interact with its environment. The concept has been established on a developed mathematical model of human cognitive-behavioural and personality psychologies.

In the presented IDS governing process, emotions play an auxiliary, though important, role of a modifier influencing the basic decision system, which is founded on an analysis of the state of the fulfilment of the system/robot needs. In brief, our emotion system is composed of three elements:

- (linear/fuzzy, classical) emotion,

- (associative/linguistic) sub-emotions,

- $\operatorname{mood}($ a derivative of emotion).

The proposed approach has the following distinguishing features:

1. External signals (stimuli) are preprocessed and definite objects are identified. 
2. Those objects and their characteristics influence attention and, in particular, emotions, mood, and needs.

3. A fuzzy reasoning framework is a piece of the thinking module.

The developed concept of an intelligent decisionmaking system is ready for complete implementation, which could govern mobile and stationary robots. Clearly, in order to get a fully autonomous system able to adapt to changing environmental conditions, any practical implementation on a mobile robot platform has to be equipped with a number of suitable sensors, an adequate processor and a sufficient amount of memory. Then it can perform its mission intelligently, and be capable of 'knowing' when it has to change battery, how to take care about itself, and under which circumstances it should execute the assigned tasks.

Further studies are intended to improve the system and its effectiveness, and, in particular, to extend its adaptivity (by solving the problem of including certain mood features, and generating new reactions, for instance). Clearly, a great challenge lies in implementation of the IDS on standard mobile robots.

Some preliminary results of a working simplified implementation of the IDS on a laboratory robot platform have also been presented. In particular, experiments concerning three scenarios of need satisfaction (illustrating the effects of the decision making system), along with the corresponding results (given in Tables 1-3), and the added piece of results of experimenting with a primitive laboratory mobile robot, having the curiosity, safety, rest, and energy needs (placed on Maslow's pyramid, from the top to the bottom levels), appear to be adequate (approximately, from a technical point of view, the robot behaved like an infant). Certainly, the primary IDS design presented here though shall be followed by further development and possibly full-blown implementation ready for vast testing conditions.

Certainly, there are a great number of works on fuzzy reasoning used for the purpose of choosing suitable behaviour. For instance, in the FLAME model (El-Nasr et al., 2000), the authors utilize the idea of fuzzy emotions; they are however directly associated to certain external events (signals), to make up the decision (the authors also solves the problem of conflicting emotions, and add some learning to incorporate some adaptation facilities). Such approaches are generally based on direct signal interpretation and filtering. Our proposition for the reasoning module can be treated as a sample/illustrative solution. It can also be founded on any of the different algorithms developed in the literature (e.g., Bui et al., 2002; Bonarini et al., 2006).

An intelligent decision-making system able to imitate human motivation could be found functional in many circumstances, from security guards, smart devices, human artificial limbs, to personal companions. A security guard can be implemented in the form of a smart dog snooping around and looking for a thread to the integrity of a given sphere. A group of such guard-bots can co-operate and obey the orders of their supervisor. In general, the perspectives of using personal companions or servants appear to be limitless.

Another sample application can be found in hand bio-manipulators, which for several reasons still look quite artificial. Note that their aesthetic and functional aspects, apart from being managed by the disabled, can perhaps be mastered by adding certain natural-like spontaneous movements being controlled by a motivation system during idleness.

\section{References}

Arons, B. (1992). A Review of the Cocktail Party Effect, MIT Media Lab., Cambridge, MA.

Biddle, S., Fox, K. and Boutcher, S. (2000). Physical Activity and Psychological Well-being, Psychology Press, London.

Blechman, E.A. (1990). Emotions and the Family: For Better or for Worse, Lawrence Erlbaum, Hillsdale, NJ.

Bonarini, A., Matteucci, M. and Restelli, M. (2006). Concepts and fuzzy models for behavior-based robotics, International Journal of Approximate Reasoning 41(2): 110-127.

Broadbent, D.E. (1958). Perception and Communication, Pergamon Press, London.

Bui, T., Heylen, D., Poel, M. and Nijholt, A. (2002). ParleE: An adaptive plan based event appraisal model of emotions, $K I$ 2002: Advances in Artificial Intelligence, Aachen, Germany, pp. 1-9.

Dewey, J. (1997). How We Think, Dover Publications, Mineola, NY.

Drachman, D.A. (2005). Do we have brain to spare?, Neurology 64(12): 2004-2005.

Ekman, P. and Davidson, R. (1999). The Nature of Emotion, Gdańskie Wydawnictwo Psychologiczne, Gdańsk, (in Polish).

El-Nasr, M.S., Yen, J. and Ioerger, T.R. (2000). FLAME—Fuzzy logic adaptive model of emotions, Autonomous Agents and Multi-agent Systems 3(3): 219-257.

Gratch, J. and Marsella, S. (2004). Evaluating a computational model of emotion, Journal of Autonomous Agents and Multiagent Systems 11(1): 23-43.

Hansson, S.O. (1994). Decision theory: A brief introduction, Technical report, Royal Institute of Technology (KTH), Stockholm.

Kahneman, D. (1973). Attention and Effort, Prentice Hall, Englewood Cliffs, NJ.

Kowalczuk, Z. and Czubenko, M. (2009). Intelligent decision system of an autonomous robot, Seminar on Robot Autonomy, Poznań, Poland. 
Kowalczuk, Z. and Czubenko, M. (2010a). DICTOBOT-An autonomous agent with the ability to communicate, Zeszyty Naukowe Wydziału ETI Politechniki Gdańskiej. Technologie Informacyjne, pp. 87-92.

Kowalczuk, Z. and Czubenko, M. (2010b). Interactive cognitivebehavioural decision making system, in L. Rutkowski (Ed.), Artificial Intelligence and Soft Computing (II), Lecture Notes in Artificial Intelligence, Vol. 6114, SpringerVerlag, Berlin/Heidelberg/New York, NY, pp. 516-523.

Kowalczuk, Z. and Czubenko, M. (2010c). Model of human psychology for controlling autonomous robots, 15th International Conference on Methods and Models in Automation and Robotics (MMAR), Międzyzdroje, Poland, pp. 31-36.

Łęski, J. (2008). Neuro Fuzzy Systems, Wydawnictwa NaukowoTechniczne, Warsaw, (in Polish).

Leite, I., Pereira, A., Martinho, C. and Paiva, A. (2008). Are emotional robots more fun to play with?, 17th IEEE International Symposium on Robot and Human Interactive Communication, RO-MAN 2008, Munich, Germany, pp. $77-82$.

Levine, D.S. (2007). Neural network modeling of emotion, Physics of Life Reviews 4(1): 37-63.

Lindsay, R.H. and Norman, D.A. (1984). Processing of Human Information, Wydawnictwo Naukowe PWN, Warsaw, (in Polish).

Maruszewski, T. (2001). Cognitive Psychology, Gdańskie Wydawnictwo Psychologiczne, Gdańsk, (in Polish).

Maslow, A. H. (1968). Toward a Psychology of Being, 2nd Edn., Van Nostrand Reinhold, New York, NY.

Mintzberg, H., Raisinghani, D. and Théorêt, A. (1976). The structure of 'unstructured' decision processes, Administrative Science Quarterly 21(2): 246-275.

Monsell, S. (1996). Control of mental processes, in V. Bruce (Ed.) Unsolved Mysteries of the Mind. Tutorial Essays in Cognition, Taylor and Francis, Hove, pp. 93-148.
Nęcka, E., Orzechowski, J. and Szymura, B. (2006). Cognitive Psychology, PWN, Warsaw, (in Polish).

Plutchik, R. (2001). The nature of emotions, American Scientist 89(4): 344.

Poggi, I., Pelachaud, C., Rosis, F., Carofiglio, V. and Carolis, B. (2005). Greta. A believable embodied conversational agent, in O. Stock and M. Zancanaro (Eds.) Multimodal Intelligent Information Presentation, Springer, Dordrecht, pp. 3-25.

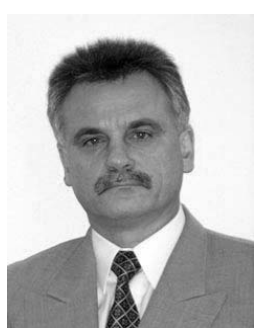

Zdzisław Kowalczuk, Prof., D.Sc., Ph.D., M.Sc.E.E. (2003, 1993, 1986, 1978). Since 1978 he has been with the Faculty of Electronics, Telecommunications and Computer Science at the Technical University of Gdańsk, where he is a full professor in automatic control and the chair of the Department of Decision Systems. He held visiting appointments at the University of Oulu (1985), Australian National University (1987), Technische Hochschule Darmstadt (1989), and George Mason University (1990-1991). His main interests include adaptive and predictive control, system identification, failure detection, signal processing, artificial intelligence, control engineering and computer science. He has authored and co-authored 10 books and 45 book chapters, about 80 journal papers and 190 conference publications. He is a recipient of 1990 and 2003 Research Excellence Awards of the Polish National Education Ministry, and a 1999 Polish National Science Foundation Award in automatic control.

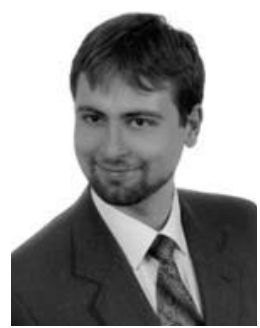

Michał Czubenko, a 2009 graduate of the Department of Decision Systems, Faculty of Electronics, Telecommunications and Informatics, Gdańsk University of Technology, in automatic control and robotics. Presently, a Ph.D. student in the same faculty, associated with the Department of Decision Systems. His interests include robotics, artificial intelligence, psychology, chess and guitar playing.

Received: 26 July 2010 Revised: 21 March 2011 